\title{
Precision measurement of the mass difference between light nuclei and anti-matter counterparts
}

\author{
Manuel Colocci* (for the ALICE Collaboration) \\ Dipartimento di Fisica dell'Università di Bologna and INFN-sezione di Bologna. Via Irnerio 46, \\ 40126 Bologna, Italy \\ E-mail: manuel.colocciebo.infn.it
}

\begin{abstract}
In ultra relativistic heavy-ion collisions a large and similar amount of nuclei and anti-nuclei is produced in the central pseudorapidity region allowing one to thoroughly investigate their properties. Mass and electric charge are expected to be the same in nuclei and anti-nuclei as long as the CPT invariance holds for nuclear force, a remnant of the underlying strong interaction between quarks and gluons. The measurements of the difference of mass-to-charge ratio between deuteron and anti-deuteron, and ${ }^{3} \mathrm{He}$ and ${ }^{3} \overline{\mathrm{He}}$ nuclei performed with the ALICE detector at the LHC are reported. The measurements improve by one to two orders of magnitude analogous results previously obtained. The results can also be expressed in terms of binding energy differences, which in the case of the (anti)-deuteron improves the constraints on CPT invariance inferred by existing measurements by a factor two. For the (anti) ${ }^{-3} \mathrm{He}$ an invariance constraint has been determined for the first time.
\end{abstract}

The European Physical Society Conference on High Energy Physics

22-29 July 2015

Vienna, Austria

${ }^{*}$ Speaker. 


\section{Introduction}

All physics laws are supposed to be the same after the application of a CPT transformation i.e. a simultaneous reversal of charge(s) (charge conjugation $\mathrm{C}$ ), reflection of spatial coordinates (parity transformation $\mathrm{P}$ ) and time inversion $(\mathrm{T})$. The $\mathrm{CPT}$ invariance is theoretically guaranteed within a description of the interactions in terms of a local quantum field theory constructed in a flat space-time, based on the Lorentz invariance and on unitarity. If some of the conditions which back-up the CPT invariance are not satisfied, the symmetry could be violated. It has been tested in various sectors i.e. for elementary fermions [3] and bosons [4], and for Quantum Electrodynamics (QED) [5] and Quantum Chromodynamics (QCD) systems [6]. Experiments are based on an important consequence of CPT symmetry, which implies that the mass, the lifetime and the absolute value of the charge and the magnetic moment of an anti-particle should be exactly the same as those of the corresponding particle. For baryons the charge-to-mass ratio of proton and anti-proton has been recently compared with a very high accuracy [6] resulting in the most stringent test of the CPT symmetry for systems bound by the strong force. The extension of such measurement from (anti)baryons to (anti-)nuclei allows one to probe any difference in the interactions between nucleons and anti-nucleons contributing to the (anti-)nuclei masses. This force is a remnant of the underlying strong interaction among quarks and gluons and can be described by effective theories [7], but cannot yet be directly derived from QCD. An efficient source of nuclei and anti-nuclei are the relativistic heavy-ion collisions at the Large Hadron Collider (LHC): a large amount of energy may be deposited in a small volume, allowing for creation of the Quark-Gluon Plasma [8] (QGP), a phase of matter in which the quark and gluon degrees of freedom normally confined within hadrons are liberated. The created system expands and cools down after its production, and the transition into a hadron gas occurs. In the final state of this process a large amount of light (anti-)nuclei is also formed [9] by a coalescence process of two or more (anti-)nucleons at the last stage of the collision and/or during the hadronization process at the chemical freeze-out of the QGP. To date, the heaviest anti-nucleus observed is ${ }^{4} \overline{\mathrm{He}}[10]$. For lighter nuclei and anti-nuclei, which are more copiously produced, a detailed comparison of their properties is possible.

In this report a precision measurement of the mass-over-charge ratio between deuteron (d) and anti-deuteron $(\overline{\mathrm{d}})$ and between ${ }^{3} \mathrm{He}$ and $(\text { anti- })^{3} \mathrm{He}$ nuclei carried out with the ALICE detector [11] in $\mathrm{Pb}-\mathrm{Pb}$ collisions at a centre-of-mass energy per nucleon pair of $\sqrt{s_{\mathrm{NN}}}=2.76 \mathrm{TeV}$ is reported.

\section{Data Analysis}

The main detectors used in the analysis are the ITS (Inner Tracking System) for the determination of the interaction-vertex position and, coupled with the TPC (Time Projection Chamber), for tracking. The TPC is also used for average specific energy loss measurements $\langle\mathrm{d} E / \mathrm{d} x\rangle$ and the TOF (Time Of Flight detector) to measure the time taken by particles to reach the detector $\left(t_{\mathrm{TOF}}\right)$. The combined ITS and TPC information is used to determine the track length $(L)$ and the rigidity $(p / z)$, where $p$ is the momentum and $z$ the electric charge in units of the elementary charge $(e)$ of the charged particles in the solenoidal $0.5 \mathrm{~T}$ magnetic field of the ALICE central barrel. Based on these measurements, the squared mass-over-charge ratio is extracted $\mu_{\mathrm{TOF}}^{2} \equiv(m / z)_{\mathrm{TOF}}^{2}=(p / z)^{2}\left[\left(t_{\mathrm{TOF}} / L\right)^{2}-1 / c^{2}\right]$. The related distribution is filled requiring also that 
the track $\langle\mathrm{d} E / \mathrm{d} x\rangle$ is compatible, within $2 \sigma$ (where $\sigma$ is the standard deviation), with the expected value for each (anti-)particle type. In this way the background originated from tracks associated to an incorrect TOF hit is strongly suppressed: down to $4 \%$ for deuterons at low rigidities (below $2 \mathrm{GeV} / \mathrm{c}$ ) and down to $1 \%$ for ${ }^{3} \mathrm{He}$ nuclei. For each of the particle species, the mass-over-charge ratio is extracted by fitting the $\mu_{\mathrm{TOF}}^{2}$ distributions in narrow rigidity and pseudorapidity intervals, using Gaussian function with an exponential tail that reflects the time signal distribution of the TOF detector.

Using mass differences, rather than masses themselves, allows to reduce the systematic uncertainties related to tracking, spatial alignment (affecting the measurement of the track rigidity and length) and time calibration. Despite that, residual effects are still present, due to imperfections in the detector alignment and the description of the magnetic field. In particular, the dominant contribution to the uncertainty of the mass difference is caused by uncertainty in the rigidity measurement which can reach up to $1 \%$. Since this contribution is mass independent, it is to a large extent suppressed by rescaling the masses obtained for (anti-)deuteron and (anti-) ${ }^{3} \mathrm{He}$ with the ratio between the (anti-)proton masses recommended by PDG [12] $\left(\mu_{\mathrm{p}(\overline{\mathrm{p}})}^{\mathrm{PDG}}\right)$ and the ones measured in the analysis presented here $\left(\mu_{\mathrm{p}(\overline{\mathrm{p}})}^{\mathrm{TOF}}\right)$, i.e. $\mu_{\mathrm{A}(\overline{\mathrm{A}})}=\mu_{\mathrm{A}(\overline{\mathrm{A}})}^{\mathrm{TOF}} \times\left(\mu_{\mathrm{p}(\overline{\mathrm{p}})}^{\mathrm{PDG}} / \mu_{\mathrm{p}(\overline{\mathrm{p}})}^{\mathrm{TOF}}\right)$. Conversely, systematic effects connected to the track-length measurement are mass dependent and cannot be completely accounted for using the above correction. However, they are expected to be symmetric for positive and negative particles when inverting the magnetic field. Any residual asymmetry is therefore indicative of remaining systematic uncertainties related to the detector conditions. In order to estimate them and keep these effects under control, both nuclei and anti-nuclei measurements are performed for two opposite magnetic field configurations and then averaged. Their half difference is taken as the estimate of this systematic uncertainty. It results in $\sim 0.7 \times 10^{-4}$ for deuterons and anti-deuterons while it is found negligible, relative to the statistical uncertainty, for ${ }^{3} \mathrm{He}$ and anti${ }^{3} \mathrm{He}$. Other sources of systematic uncertainties are evaluated by varying energy loss corrections applied to the reconstructed momentum, the range and the shape of the background function assumed in the fit of the $\mu_{\mathrm{TOF}}^{2}$ distributions and the track selection criteria; more details can be found in [13].

\section{Results}

The mass-over-charge ratio differences between nuclei and anti-nuclei for deuteron and ${ }^{3} \mathrm{He}$ are evaluated as a function of the rigidity of the tracks. The final result is computed as weighted average of the measurements obtained in each rigidity interval. Their statistical and uncorrelated systematic uncertainties are used as the weights. The final systematic uncertainty is then the sum in quadrature of the correlated and uncorrelated systematic uncertainties. The measured mass-over-charge ratio differences are

$$
\begin{array}{r}
\Delta \mu_{\mathrm{d} \overline{\mathrm{d}}}=[1.7 \pm 0.9(\text { stat. }) \pm 2.6(\text { syst. })] \times 10^{-4} \mathrm{GeV} / c^{2}, \\
\left.\Delta \mu_{3 \mathrm{He}^{3} \overline{\mathrm{He}}}=[-1.7 \pm 1.2 \text { (stat. }) \pm 1.4(\text { syst. })\right] \times 10^{-3} \mathrm{GeV} / c^{2},
\end{array}
$$

corresponding to

$$
\left.\frac{\Delta \mu_{\mathrm{d} \overline{\mathrm{d}}}}{\mu_{\mathrm{d}}}=[0.9 \pm 0.5 \text { (stat. }) \pm 1.4(\text { syst. })\right] \times 10^{-4},
$$




$$
\left.\left.\frac{\Delta \mu_{3^{3} \mathrm{He}^{3} \overline{\mathrm{He}}}}{\mu_{{ }_{3} \mathrm{He}}}=[-1.2 \pm 0.9 \text { (stat. }) \pm 1.0 \text { (syst. }\right)\right] \times 10^{-3},
$$

where $\mu_{\mathrm{d}}$ and $\mu_{3} \mathrm{He}$ are the values recommended by CODATA [14]. The mass-over-charge differences are compatible with zero within the estimated uncertainties, in agreement with CPT conservation. These results (Fig. 1, left) represent the highest precision direct measurements of mass differences in the sector of nuclei and they improve by one to two orders of magnitude analogous results originally obtained more than 40 years ago $[15,16,17]$. The result for the (anti-)deuteron is reported in Fig. 2 (in red), which summarizes the best CPT invariance tests obtained to date for particles, nuclei and atoms [3, 4, 5, 12, 18, 19]. These measurements can be used to constrain, for different interactions, the parameters of effective field theories with explicit CPT violating terms to the Standard Model Lagrangian, such as the Standard Model Extension [20].

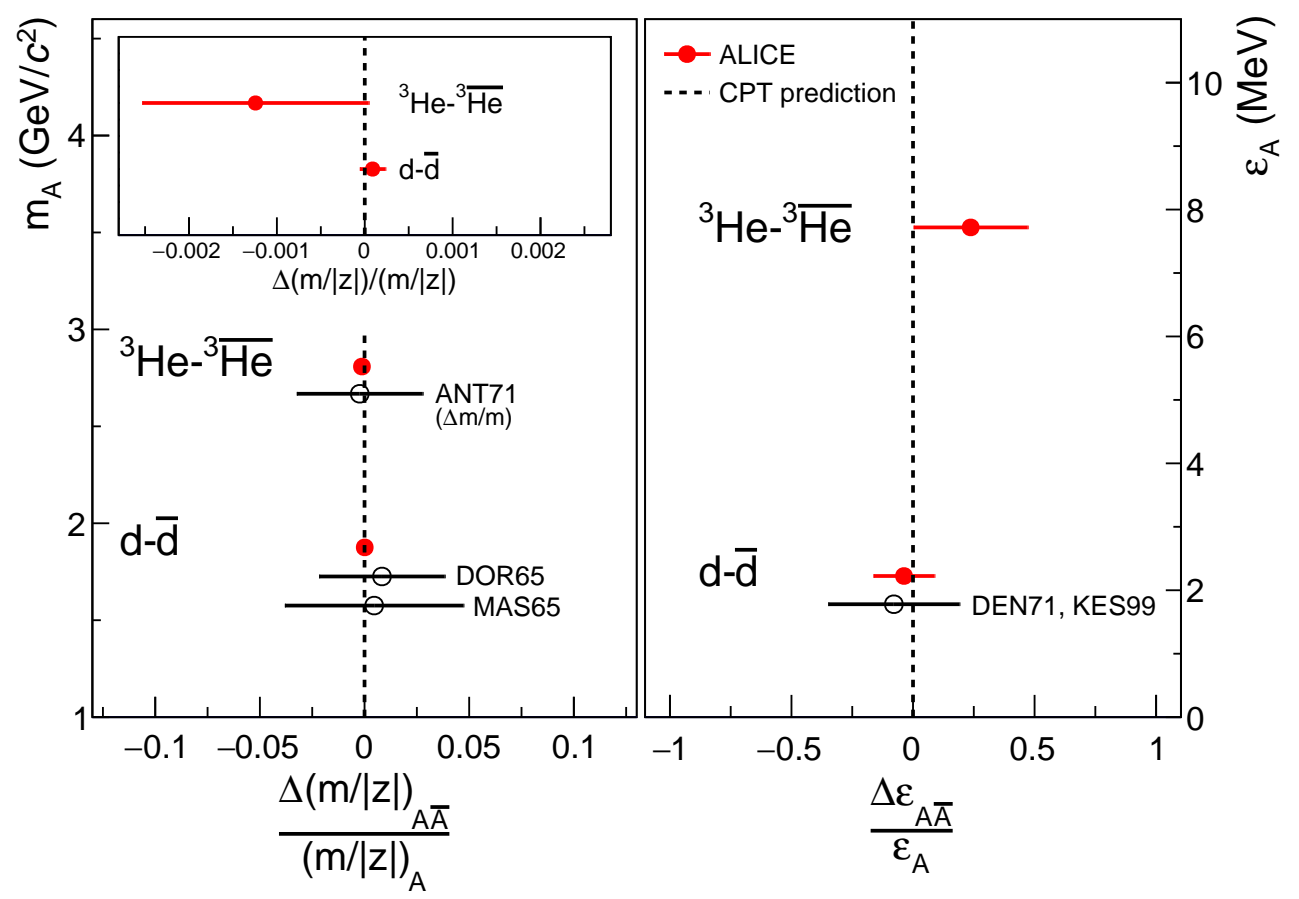

Figure 1: The ALICE measurements for $\mathrm{d}-\overline{\mathrm{d}}$ and ${ }^{3} \mathrm{He}^{-}{ }^{3} \mathrm{He}$ mass-over-charge ratio differences compared with CPT invariance expectation (dotted lines) and existing mass measurements MAS65 [15], DOR65 [16] and ANT71 [17] (left panel). The inset shows the ALICE results on a finer $\Delta(m / z) /(m / z)$ scale. The right panel shows our determination of the binding energy differences compared with direct measurements from DEN71 [22] and KES99 [23]. Error bars represent the sum in quadrature of the statistical and systematic uncertainties (standard deviations).

Given that $z_{\overline{\mathrm{d}}}=-z_{\mathrm{d}}$ and $z_{3} \overline{\mathrm{He}}=-z_{3} \mathrm{He}$ as for the proton and anti-proton [18], the mass-overcharge ratio differences in Eq. 3.1 and Eq. 3.2 and the measurement of the mass differences between proton and anti-proton [18] and between neutron and anti-neutron [21] are used to derive the 
relative binding energy difference between each nucleus and the corresponding anti-nucleus:

$$
\begin{gathered}
\frac{\Delta \varepsilon_{\mathrm{d} \overline{\mathrm{d}}}}{\varepsilon_{\mathrm{d}}}=-0.04 \pm 0.05 \text { (stat.) } \pm 0.12 \text { (syst.), } \\
\frac{\Delta \varepsilon_{3^{3} \mathrm{He}^{3} \overline{\mathrm{He}}}}{\varepsilon_{3} \mathrm{He}}=0.24 \pm 0.16 \text { (stat.) } \pm 0.18 \text { (syst.), }
\end{gathered}
$$

where $\varepsilon_{A}=Z m_{\mathrm{p}}+(A-Z) m_{\mathrm{n}}-m_{A}$, being $m_{\mathrm{p}}$ and $m_{\mathrm{n}}$ the proton and the neutron mass values recommended by PDG [12] and $m_{A}$ the mass value of the nucleus with atomic number $Z$ and mass number $A$, recommended by CODATA [14]. The binding energy is more explicitly connected with possible violations of the CPT symmetry in the (anti-)nucleon interaction from the one connected to the (anti-)nucleon masses, the latter being constrained with a precision of $7 \times 10^{-10}$ for the proton/anti-proton system [18]. The results improve (Fig. 1, right) by a factor two the constraints on CPT invariance inferred by existing measurements [22, 23] in the (anti-)deuteron system. The binding energy difference is determined for the first time in the case of (anti-) $)^{3} \mathrm{He}$, with a relative precision comparable to the one obtained in the (anti-)deuteron system.

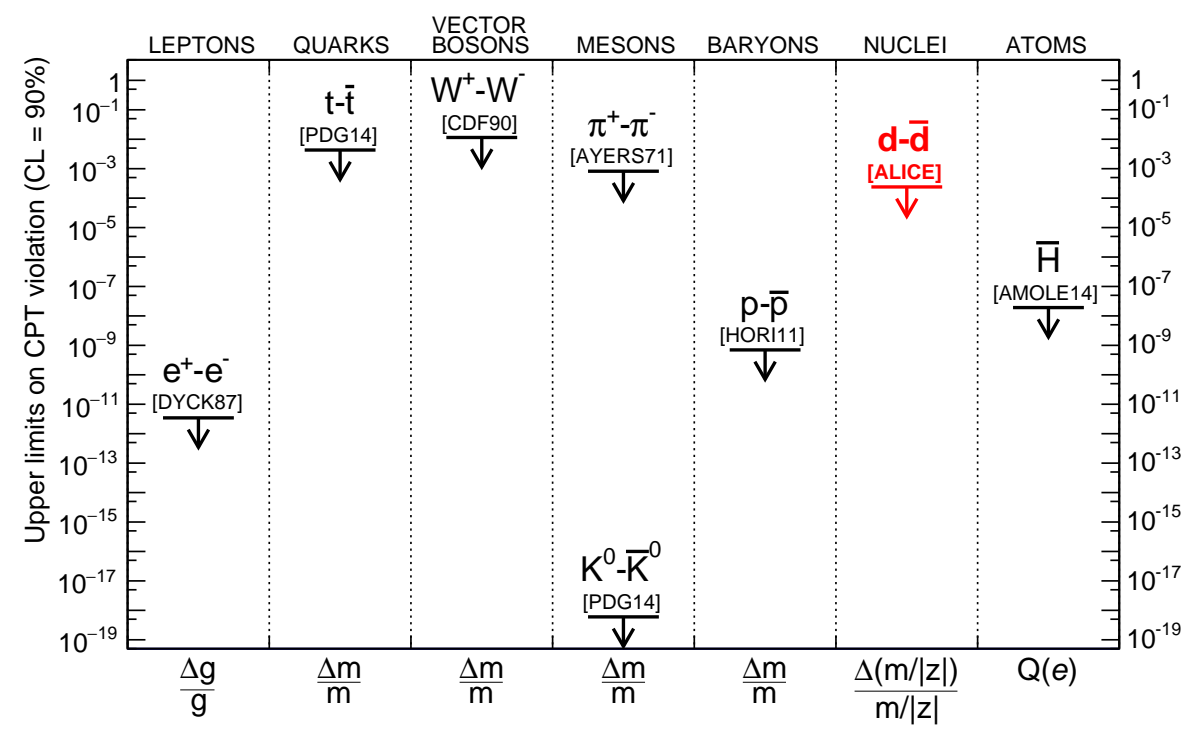

Figure 2: Experimental limits for $\mathrm{CPT}$ invariance $(\mathrm{CL}=90 \%)$ for particles, nuclei and atoms. From left to right: measurement of $\mathrm{g}$-factor for the electron and positron (DYCK87 [3]), mass difference between top and anti-top (PDG average [12]), $\mathrm{W}^{+}-\mathrm{W}^{-}$(CDF90 [4]), $\pi^{+}-\pi^{-}$(AYERS71 [19]), $\mathrm{K}^{0}-\overline{\mathrm{K}}^{0}$ (PDG average [12]), proton and anti-proton (HORI11 [18]), our result on deuteron and anti-deuteron (in red) and the charge of anti-hydrogen (AMOLE14 [5]).

\section{References}

[1] G. Lüders, On the Equivalence of Invariance under Time Reversal and under Particle-Antiparticle Conjugation for Relativistic Field Theories, Kong. Dan. Vid. Sel. Mat. Fys. Med. $28 N 5$ (1954) 1. 
[2] W. Pauli, Exclusion Principle, Lorentz Group and Reflection of Space-Time and Charge in Niels Bohr and the Development of Physics, Pergamon Press, New York 1955.

[3] R. S. Jr. Van Dyck, P. B. Schwinberg and H. G. Dehmelt, New High-Precision Comparison of Electron and Positron g Factors, Phys. Rev. Lett. 59 (1987) 26.

[4] F. Abe et al. (CDF Collaboration), A Measurement of the W-boson mass, Phys. Rev. Lett. 65 (1990) 2243.

[5] C. Amole et al., An experimental limit on the charge of antihydrogen, Nature Commun. 5 (2014) 3955.

[6] S. Ulmer et al., High-precision comparision of the antiproton-to-proton charge-to-mass ratio, Nature 524 (2015) 196.

[7] U. van Kolck, Effective field theory of nuclear forces, Prog. Part. Nucl. Phys. 43 (1999) 337.

[8] P. Braun-Munzinger and J. Stachel, The quest for the quark-gluon plasma, Nature 448 (2007) 302.

[9] A. Jaroslav et al. (ALICE Collaboration), Production of light nuclei and anti-nuclei in $\mathrm{pp}$ and $\mathrm{Pb}-\mathrm{Pb}$ collisions at LHC energies, arXiv:1506.08951 [nucl-ex].

[10] H. Agakishiev et al. (STAR Collaboration), Observation of the antimatter helium-4 nucleus, Nature 473 (2011) 353.

[11] B. I. Abelev et al. (ALICE Collaboration), Performance of the ALICE experiment at the CERN LHC, Int. J. Mod. Phys. A 29 (2014) 1430044.

[12] K. A. Olive et al. (Particle Data Group Collaboration), Review of Particle Physics, Chin. Phys. C 38 (2014) 090001.

[13] J. Adam et al. (ALICE Collaboration), Precision measurement of the mass difference between light nuclei and anti-nuclei, Nature Phys. 11 (2015) 811, ALICE-PUBLIC-2015-002.

[14] P. J. Mohr, B. N. Taylor and D. B. Newell, CODATA Recommended Values of the Fundamental Physical Constants: 2010, Rev. Mod. Phys. 84 (2012) 1527.

[15] T. Massam, Th. Muller, B. Righini, M. Schneegans and A. Zichichi, Experimental observation of antideuteron production, Nuovo Cimento 39 (1965) 10.

[16] D. E. Dorfan, J. Eades, L. M. Lederman, W. Lee and C. C. Ting, Observation of antideuterons, Phys. Rev. Lett. 14 (1965) 1003.

[17] Yu. M. Antipov et al., Observation of antihelium-3, Nucl. Phys. B 31 (1971) 235.

[18] M. Hori et al., Two-photon laser spectroscopy of antiprotonic helium and the antiproton-to-electron mass ratio, Nature 475 (2011) 484.

[19] D. S. Ayers et al., Measurements of the lifetimes of positive and negative pions, Phys.Rev. D3 (1971) 1051.

[20] V. A. Kostelecký, N. Russel, Data tables for Lorentz and CPT violation, Rev. Mod. Phys 83 (2011) 11.

[21] M. Cresti, G. Pasquali, L. Peruzzo, C. Pinori and G. Sartori, Measurement Of The Anti-neutron Mass, Phys. Lett. B 177 (1986) 206, Errata, Phys. Lett. B 200 (1988) 587.

[22] S. P. Denisov et al., Measurements of anti-deuteron absorption and stripping cross sections at the momentum $13.3 \mathrm{GeV/c}$, Nucl. Phys. B 31 (1971) 253.

[23] E. G. Jr. Kessler et al., The Deuteron Binding Energy and the Neutron Mass, Phys. Lett. A 255 (1999) 221. 